\title{
Cognitive behavioural therapy reduces short term rehospitalisation compared with psychoeducation in inpatients with schizophrenia
}

Bechdolf A, Knost B, Kuntermann C, et al. A randomized comparison of group cognitive-behavioural therapy and group psychoeducation in patients with schizophrenia. Acta Psychiatr Scand 2004;1 10:21-8.

How does group cognitive behavioural treatment compare with psychoeducation for inpatients with schizophrenia?

\section{METHDS}

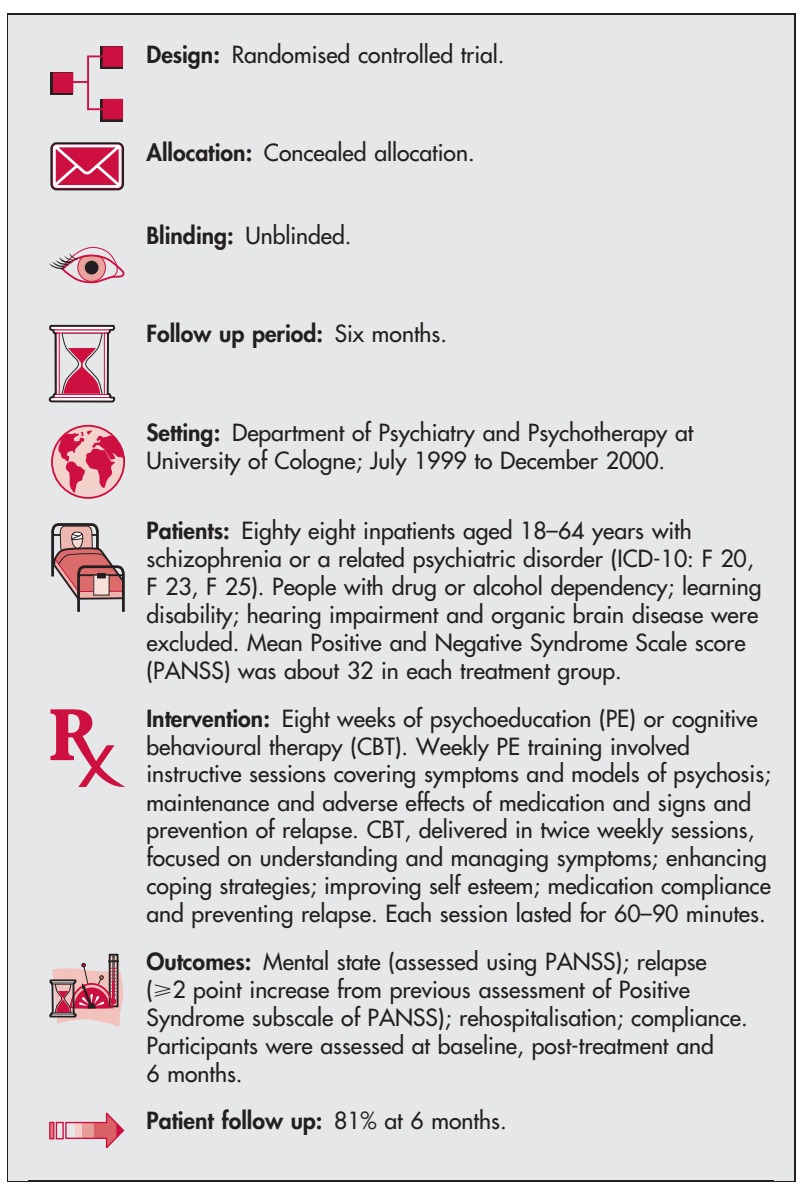

\section{MAIN RESULTS}

CBT reduced hospitalisation rates compared with PE at 6 months $(0.0 \%$ with CBT $v 12.5 \%$ with PE; $\mathrm{p}=0.04)$. Medication compliance was similar in both treatment groups $(\mathrm{p}=0.27$ at 6 months). However, there were no significant differences in mental state between treatment groups at 6 month follow up (mean PANSS 28.5 with CBT $v 26.0$ with PE; $\mathrm{p}=0.27$ ). There was no significant difference in relapse between groups (13\% with CBT v 20\% with PE; $\mathrm{p}=0.43$ )

For correspondence: Andreas Bechdolf, Department of Psychiatry and Psychotherapy, University of Cologne, 50924 Cologne, Germany; andreas.bechdolf@medizin.uni-koeln.de

Sources of funding: Koln Fortune Program grant, Faculty of Medicine, University of Cologne.

\section{CONCLUSIONS}

A brief CBT intervention for inpatients with schizophrenia reduces rehospitalisation rates compared with psychoeducation.

\section{NOTES}

Patients were randomised in blocks of eight for CBT or PE treatment. Patients in the CBT group received an average of 11.9 sessions (of a maximum 16 sessions) while those in the PE group received an average of 6.4 sessions (of a maximum 8 sessions). Contact time was not controlled for in the analysis. Conclusions may be limited by small sample size.

\section{Commentary}

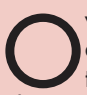
ver the past 15 years, there has been significant development and refinement of cognitive behavioural therapy (CBT) interventions for people experiencing persistent symptoms of schizophrenia. While the preponderance of randomised controlled trials have tested the additive benefit of CBT to standard care for people in the chronic phase of the illness, newer studies have focused on potential benefits in the acute phase of the illness, preventing relapse, and as a combined intervention for targeting psychosis and existing comorbidities such as substance dependence. Previous meta-analytic reviews of the literature have concluded that CBT in combination with standard care (for example, medications and case management) results in significant reductions of positive and negative symptoms. ${ }^{1}{ }^{2}$ A more recent metaanalysis of 20 RCTs (a total of 739 patients) arrived at a similar conclusion, suggesting good evidence for the efficacy and effectiveness of CBT for schizophrenia. ${ }^{3}$ In summary, there is mounting support for the evidence-based practice of CBT for schizophrenia.

The vast majority of studies have been conducted in the UK and so there is continuing need to address the feasibility, efficacy, and effectiveness of CBT for schizophrenia in the treatment contexts of other healthcare systems. This study by Bechdolf et al conducted in Germany, addresses the efficacy of a structured, 8 week CBT protocol (16 sessions) for patients in the acute phase of the illness. Although patients treated with CBT versus psychoeducation showed lower rates of rehospitalisation over the follow up phase, there were no between-group differences during the acute or follow up with respect to symptom reduction. The ability of CBT to impact on a clinically meaningful outcome variable such as rehospitalisation following the acute phase is extremely meaningful. However, this study also leaves us with the important question as to whether standard CBT protocols require refinement when the timing of intervention shifts from the chronic to the acute phase of the illness. Neil A Rector, PhD, CPsych Mood and Anxiety Program, Centre for Addiction and Mental Health, Associate Professor of Psychiatry, University of Toronto, Toronto, Ontario, Canada

1 Gould RA, Mueser KT, Bolton E, et al. Cognitive therapy for psychosis in schizophrenia: An effect size analysis. Schizophr Res 2001;48:335-42.

2 Rector NA, Beck AT. Cognitive behavioral therapy for schizophrenia: An empirical review. J Nerv Ment Dis 2001;189:278-87.

3 Tarrier N, Wykes T. Is there evidence that cognitive behaviour therapy is an effective treatment for schizophrenia? A cautious or cautionary tale? Behav Res Ther 2004;42:1377-401. 